\title{
Exploiting simultaneous multi-frequency observations to probe polar-cap processes
}

\author{
Yogesh Maan \\ ASTRON, Netherlands Institute for Radio Astronomy \\ Postbus 2, 7990 AA Dwingeloo, the Netherlands \\ email: maan@astron.nl
}

\begin{abstract}
Sub-pulse drifting has been regarded as one of the most insightful aspects of the pulsar radio emission. The phenomenon is generally explained with a system of emission sub-beams rotating around the magnetic axis, originating from a carousel of sparks near the pulsar surface (the carousel model). Since the observed radio emission at different frequencies is generated at different altitudes in the pulsar magnetosphere, corresponding sampling of the carousel on the polar cap differs slightly in magnetic latitude. When this aspect is considered, it is shown here that the carousel model predicts important observable effects in multi-frequency or wide-band observations. Also presented here are brief mentions of how this aspect can be exploited to probe the electrodynamics in the polar cap by estimating various physical quantities, and correctly interpret various carousel related phenomena, in addition to test the carousel model itself.
\end{abstract}

Keywords. pulsars: general, radiation mechanisms: general

\section{Carousel model and radius-to-frequency mapping of radio emission}

A considerable fraction of pulsars exhibit a systematic modulation of intensity within individual pulses. The modulation appearing in the form of components within single pulses, i.e., sub-pulses, generally drifts in pulsar's rotation phase from pulse-to-pulse. This fascinating phenomenon, called sub-pulse drifting, is generally attributed to the presence of a system of emission sub-beams stemmed from a carousel of 'sparks' near the pulsar surface rotating around the magnetic axis (the carousel model; Ruderman \& Sutherland 1975).

Radius-to-frequency mapping (RFM; Cordes 1978) suggests the observed radio emission at different frequencies to be generated at different altitudes in the pulsar magnetosphere. Furthermore, foot-points of the field lines that give rise to the emission at different altitudes have slightly different magnetic colatitudes at the polar cap. Thus, different frequencies sample slightly offset slices of the polar cap, and hence, the carousel at its different rotation phases. This aspect implies important observable signatures of the carousel in multi-frequency (e.g., Maan et al. 2013) and wide-band observations, as briefed below.

\section{Multi-frequency observational consequences}

To demonstrate the observational effect of the above aspect at different frequencies, two pulse-sequences were generated (at presumed frequencies of 240 and $610 \mathrm{MHz}$ ) from a simulated carousel of 6 sparks and using an empirical relationship for RFM. For a sightline non-tangential to the emission beam, such a carousel would produce two pulsecomponents. The different sampling of the carousel at its slightly different circulation phases reflects as an offset in sub-pulse modulation phase under a given pulse-component at different frequencies. Moreover, such a phase offset will be equal in magnitude but 

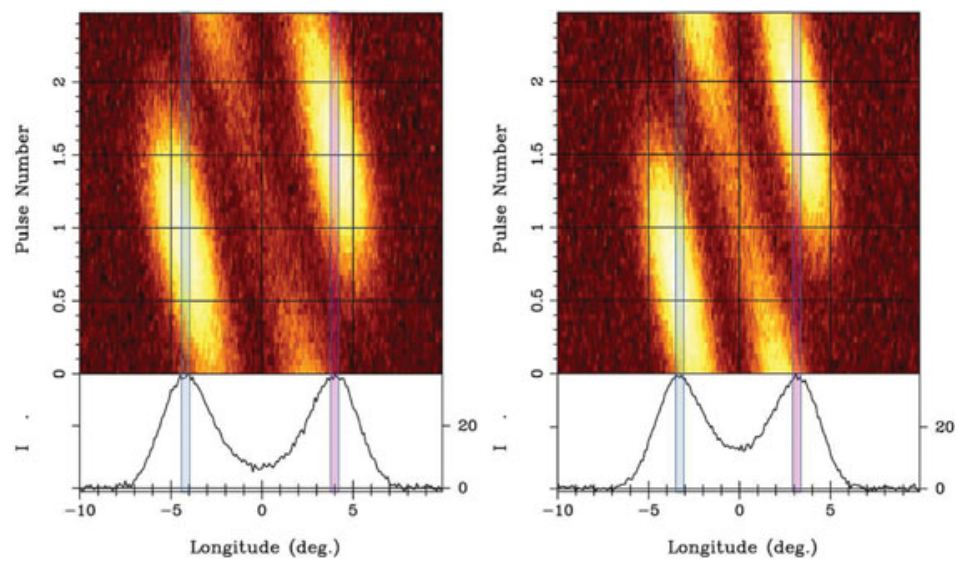

Figure 1. Example of two pulse-sequences simulated at 240 and $610 \mathrm{MHz}$ and folded over the sub-pulse modulation period. The offset in sub-pulse modulation phase under the corresponding components (compare, for example, the light blue shaded regions) is clearly evident.

opposite in direction for the two components (see Figure 1). The magnitude of the phase offset is primarily contributed by the difference in magnetic azimuths corresponding to the pulse-longitudes of the given pulse-component at different frequencies. A relatively smaller, second order contribution comes from the circulation of the carousel during the time corresponding to the difference in pulse-longitudes of the same component at different frequencies.

For a known viewing geometry and measurable sub-pulse modulation period, $\mathrm{P}_{3}$, the above offset in sub-pulse phase is a completely deterministic quantity and can be exploited to determine the carousel circulation period, potentially resolve the $\mathrm{P}_{3}$-aliasing issue even for non-tangential sightlines, and test the carousel model itself using simultaneous dual/multi-frequency observations. The above phase offset, when translated to the corresponding delay, can be further exploited to directly measure the angular velocity of the carousel using the pulse intensity fluctuations observed simultaneously at two or more radio frequencies, even if no sub-pulse modulation is apparent (due to, e.g., an irregular or unstable carousel). This phase-offset also has important implications when interpreting multi-frequency occurrences of pseudo-nulls, sub-pulse modulations in orthogonal polarization modes, exploring any twist in the field line geometry using sub-pulse modulations (Maan \& Deshpande 2014), etc.

Summarizing, the carousel model along with the RFM predicts offsets in sub-pulse modulation phases under a given pulse-component when observed simultaneously at different frequencies for viewing geometries non-tangential to the emission beam.

\section{Acknowledgements}

The research leading to these results has received funding from the European Research Council under the European Union's Seventh Framework Programme (FP/2007-2013) / ERC Grant Agreement n. 617199.

\section{References}

Cordes, J. M. 1978, ApJ, 222, 1006

Maan, Y., Deshpande, A. A., Chandrashekar, V., et al. 2013, ApJS, 204, 12

Maan, Y. \& Deshpande, A. A. 2014, ApJ, 792, 130

Ruderman, M. A. \& Sutherland, P. G. 1975, ApJ, 196, 51 